\title{
PathosisGAN: Sick Face Image Synthesis with Generative Adversarial Network
}

\author{
Jinyu $\mathrm{Hu}$ \\ Institute of Intelligent Machines, \\ Chinese Academy of Sciences, \\ University of Science and Technology \\ of China, Hefei, China \\ jinyuhu@mail.ustc.edu.cn
}

\author{
Yuchen Ren \\ Institute of Intelligent Machines, \\ Chinese Academy of Sciences, \\ University of Science and Technology \\ of China, Hefei, China \\ ycren@mail.ustc.edu.cn
}

\author{
Yuan Yuan \\ Institute of Intelligent Machines, \\ Chinese Academy of Sciences, Hefei, \\ China \\ yuanyuan@iim.ac.cn
}

\author{
Yin Li \\ Institute of Software Application \\ Technology, Chinese Academy of \\ Sciences, Guangzhou, China \\ liyin@gz.iscas.ac.cn
}

\author{
Lei Chen \\ Institute of Intelligent Machines, \\ Chinese Academy of Sciences, Hefei, \\ China, chenlei@iim.ac.cn, \\ Corresponding author
}

\section{KEYWORDS}

Generative adversarial networks, image-to-image translation, augmentation, face synthesis

\section{ACM Reference Format:}

Jinyu Hu, Yuchen Ren, Yuan Yuan, Yin Li, and Lei Chen. 2021. PathosisGAN: Sick Face Image Synthesis with Generative Adversarial Network. In 2021 2nd International Conference on Artificial Intelligence and Information Systems (ICAIIS '21), May 28-30, 2021, Chongqing, China. ACM, New York, NY, USA, 6 pages. https://doi.org/10.1145/3469213.3470691

\section{INTRODUCTION}

In the diagnostics of traditional Chinese medicine, some facial features can reflect human organs' possible lesions and abnormalities. The extensive application of deep learning technology in face recognition [1] and object detection [2] provides strong technical support for the medical analysis method based on the facial sick features detection and recognition. However, deep neural networks rely on large-scale training data to learn generalized expressive power and avoid overfitting. Unfortunately, there are few open face image datasets used in the medical field. It is challenging to collect sick face images on a large scale, which requires a lot of workforce and material resources. On the other hand, a conventional face dataset [3] is not ideal for sick features detection and recognition. Besides, the selfie camera filters, makeup modification, and low resolution make some facial subtle sick features less obvious. Therefore, face dataset has an oversampling problem caused by class imbalance, which brings great difficulties to the facial sick features detection and recognition.

The success of Generative Adversarial Networks [4] based imageto-image translation methods have made sick face image synthesis possible. Traditional image-to-image translation methods based on GAN (such as Pix2Pix [5]) require paired training data, which have great limitations in practical applications. Recently, the image translation method with unpaired training data has achieved great results. CycleGAN [6], DiscoGAN [7], and DualGAN [8] achieve unpaired image-to-image translation by introducing a new loss function called cycle consistency constraint. However, it is not feasible to directly apply the above methods in our tasks. The main reasons are as follows: 


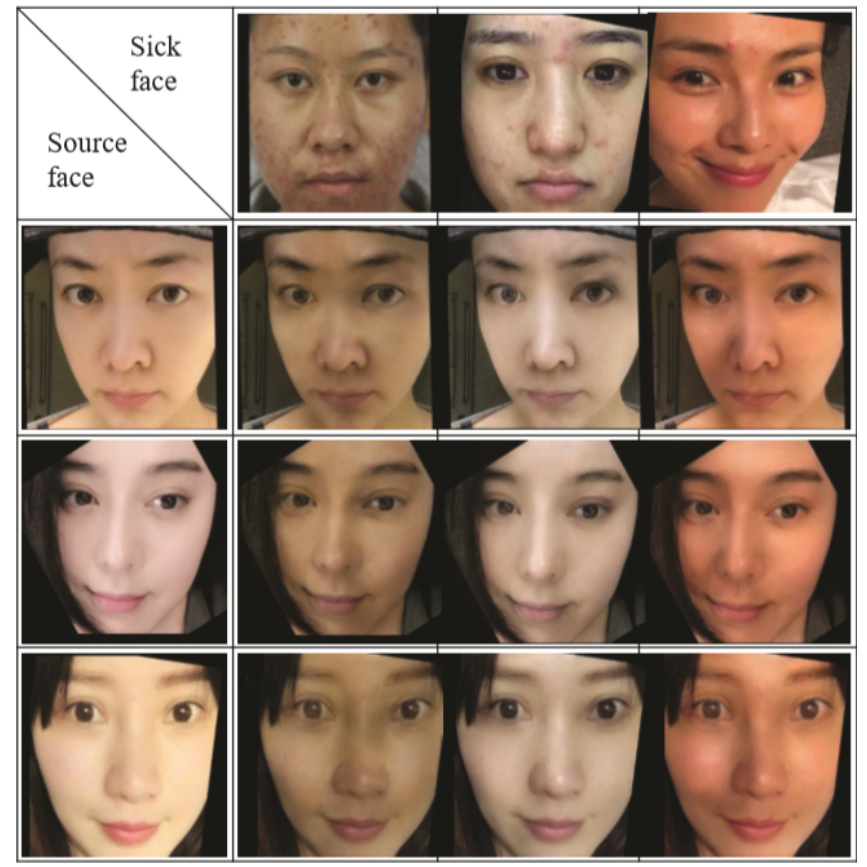

Figure 1: Results of our model for sick face image synthesis. The top row shows the silk face images. The left column shows the source images. Nine generated images are shown in the middle.

- Most existing image-to-image translation methods realize global style feature transfer. Therefore, it is difficult to extract the subtle sick features (e.g., dark circles) in the local area of human face.

- The number of images generated by image translation is limited by most methods of global feature extraction. The synthesized dataset has redundancy, which is not conducive to expanding data on a large scale.

In order to solve the above problems, we propose a GAN model called PathosisGAN. This model takes a source image and a sick face image as input, and then transfers the sick face image's sick features to the source image. Meanwhile, the primary information of the source image is retained. Based on the framework of CycleGAN, our model utilizes the mask control module to generate the face index mask. The model achieves the sick features transfer by adopting a pixel-level histogram loss calculated on local facial regions while restricting the area that does not require editing. In addition, the model can independently edit a certain facial area to realize the transfer of specific sick features. As shown in Figure 1, the generated images are true to nature with noticeable dark circles and dull complexion. We also add a feature extraction module to the generator to extract feature tensors from the feature map and synthesize face images with different degrees of sickness by adjusting the coefficient $\alpha$. In general, the main contributions of this paper are as follows:
- Based on the framework of CycleGAN, we propose a GAN model called PathosisGAN to achieve sick face image synthesis with unpaired training data. Experiments present that PathosisGAN can generate visually realistic results that provide enough medicine analysis samples based on sick face images. The mask control module is introduced to achieve the sick features transfer from the local facial area of sick face images to the source image while keeping the source image's primary information unchanged.

- We add a feature extraction module to the generator to extract feature tensors from the input images and synthesize face images with different degrees of sickness, further strengthening the data augmentation effect of sick face images.

\section{RELATED WORK}

\subsection{Generative Adversarial Networks}

Generative Adversarial Networks [4] was initially proposed by Ian Goodfellow et al, which uses adversarial networks to train the generative network's architecture to learn the real data distribution. The key to the success of GAN is to propose the concept of adversarial loss, which makes the generated image indistinguishable from the real image in principle. Therefore, GAN has been widely used in computer vision tasks such as image generation and image editing. [9] proposed a deep Convolutional Neural Network (CNN) model for the transfer of face attributes and retained the input source images' identity information. [10] edited the face attributes by learning the corresponding residual images. [11] proposed a conditional adversarial autoencoder to realize the age progression and regression of face images at the same time.

\subsection{GAN for Image-to-Image Translation}

Image translation usually transforms the image from the source domain to the target domain. The translated image contains some features of the target domain and retains the original attributes. In recent years, the application of GAN in this field has achieved remarkable results. Pix2pix [5] proposes a paired data image translation method based on the CGAN [12] framework. The Perceptual Adversarial Network (PAN) [13] introduces the perceptual loss into the generative adversarial loss under paired data. It uses the difference between the input image and the real image in the discriminator's hidden layer. It minimizes the difference in perceptual information from the discriminator, making the converted image have perceptual similarity. CycleGAN [6] and DiscoGAN [7] realize image translation under unpaired data by introducing cycle consistent loss. Kim et al. [14] add attribute consistency loss to make the converted image have the reference image's target attributes.

\subsection{GAN for Data Augmentation}

As a method of increasing the scale of data and improving the quality of data, data augmentation technology helps to build better deep learning models. At present, GAN-based data augmentation methods [15] are mostly used in the field of biomedical image analysis $[16,17]$. Frid-Adar et al. [18] apply GAN-based data augmentation technology to liver pathological changes' classification tasks. The experimental results show that the GAN-based data augmentation 


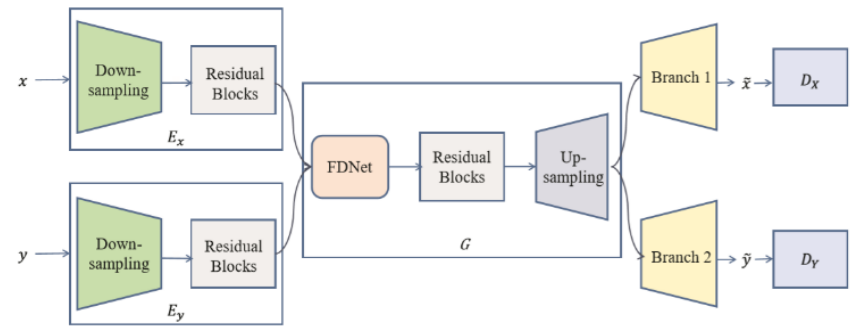

Figure 2: Overall structure of the proposed PathosisGAN. FDNet extracts the facial features from the sick face image $x, y$ : source image and sick face image. $\tilde{x}, \tilde{y}$ : the generated results.

method better improves classification performance. We can also extend data augmentation methods from image recognition to object detection tasks. Huang et al. [19] propose AugGAN, a structureperceptual image-to-image translation network. Experiments have shown that the detection accuracy has been significantly improved. Li et al. [13] generate a realistic high-resolution detection object through the adversarial branch and perception branch of the discriminator to solve the low resolution of small objects in small object detection. Ehsani et al. [20] propose a framework consisting of a segmentor, generator, and discriminator to detect and reconstruct objects occluded by other objects.

\section{PATHOSISGAN}

\subsection{Framework}

Our task is to transfer the sick face images' sick features to the source images under unpaired data, thereby synthesizing new sick face images. The non-sick face image data domain and the sick face image data domain is denoted by $X$ and $Y$, respectively. Then, we let $\left\{x^{n}\right\}_{n=1, \ldots, N}, x^{n} \in X,\left\{y^{m}\right\}_{m=1, \ldots, M}, y^{m} \in Y$ respectively represent samples of two data domains, where $x \sim p_{\text {data }}(x), y \sim p_{\text {data }}(y)$. For a given source image $x$ and a given sick face image $y$, our proposed PathosisGAN needs to learn the mapping between the two data domains, denoted as $G:\{x, y\} \rightarrow\{\tilde{x}, \tilde{y}\}$. The generated image $\tilde{x}$ contains the sick features of the sick face image $y$ while retaining the primary information of the source image. $\tilde{y}$ realizes sick facial features removal from $y$. The network structure shown in Figure 2 contains two encoders $E_{x}$ and $E_{y}$, a generator $G:\{x, y\} \rightarrow\{\tilde{x}, \tilde{y}\}$, two output branches Branch1 and Branch2, two discriminators $D_{x}$ and $D_{y}$ for data domain $X$ and data domain $Y$ respectively.

Moreover, we add a feature extraction module called FDNet to the generator $G$, and feed the feature maps $V_{x} \in \mathbb{R}^{C \times H \times W}$ and $V_{y} \in \mathbb{R}^{C \times H \times W}$ obtained from the two encoders to two $1 \times 1$ convolutional layer, which is adopted to extract the various facial features extracted by the encoder into two feature matrixes $\psi \in \mathbb{R}^{1 \times H \times W}$ and $\omega \in \mathbb{R}^{1 \times H \times W}$, and repeatedly expand the feature matrixes along the number of channels into feature tensors $\psi \in \mathbb{R}^{C \times H \times W}$ and $\omega \in \mathbb{R}^{C \times H \times W}$, where $C, H, W$ respectively represent the number of channels, the height and width of the feature map. The activation value of the generated feature map $V_{x}{ }^{\prime}$ can be expressed as:

$$
V_{x}^{\prime}=\left(\alpha \Psi_{x}+(1-\alpha) \Psi_{y}\right) V_{x}+\left(\alpha \Omega_{x}+(1-\alpha) \Omega_{y}\right)
$$

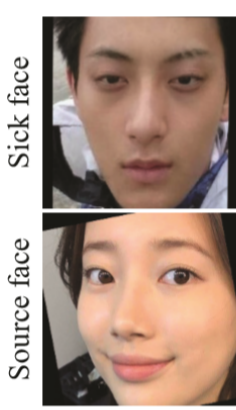

Image

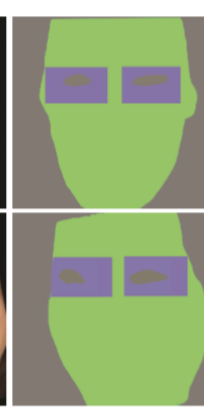

M

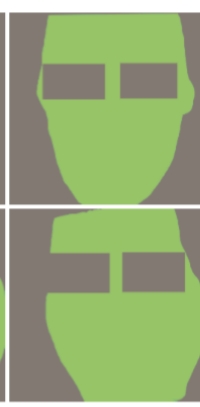

$M_{\text {skin }}$

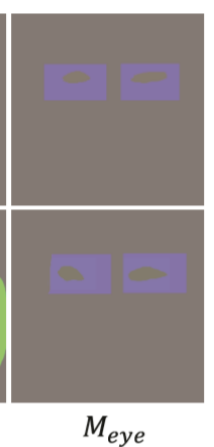

Figure 3: Given the sick face (1st row) and the source face (2nd row), the face masks are generated by a mask generation network. The second column are the index masks to represent each facial area. The third column are the binary masks corresponding to the eye area. The fourth column are the binary masks corresponding to the facial skin area.

where the coefficient $\alpha \in[0,1]$ is used to control the sickness degree of the sick features transferred in the generated image.

\subsection{Mask Control Module}

Traditional image-to-image translation methods often change the global features of the entire image. In contrast, the face's sick features are subtle in the face's local area and need to be edited independently for the eye area and facial skin areas. Meanwhile, other irrelevant areas should be consistent with the source image. In order to tackle this problem, we realize the transfer of sick features on the local area of the face through the mask control module. At the same time, we restrain the hair, background and other areas from changing significantly. In addition, we can independently edit a certain face area to achieve specific sick features transfer.

In order to obtain a face mask, we introduce a mask generation network. The mask generation network is a face Semantic Segmentation Network, which obtains the face mask $M=F P(x)$ (the second column in Figure 3) by segmenting the face image. For a given face image, the mask generation network will generate an index mask $M$ to represent each facial area, including: (1) Facial skin area, which refers to the skin area of the face excluding the lip area and the eye area, which can be used for the transfer of dull complexion; (2) Eye area, refers to the skin below the eyebrows and around the eyes. We calculate two rectangular areas covering the skin around the eyes, and then exclude areas such as eyes, hair, and eyebrows. It can be used for the transfer of dark circles and eye bags; (3) Invariable area, refers to other areas that have nothing to do with sick features, including background, hair, ears, neck, teeth. For the facial skin area and the eye area, we generate two corresponding binary masks: $M_{\text {skin }}$ (the third column in Figure 3) and $M_{\text {eye }}$ (the fourth column in Figure 3).

The transfer of sick features of the human face can actually be seen in the mapping of the color distribution of the corresponding region of the source image and the sick face image. We use pixellevel local histogram loss to realize the color transformation of the corresponding region of the face. For calculating the pixel-level 
histogram loss between the source image $\mathrm{x}$ and the sick face image $y$, we first need to match the histograms of $x$ and $y$ to obtain a recombined image $H M(x, y)$. The image has the same color distribution as $y$ and retains the primary information of $x$. Then we use MSE loss between $H M(x, y)$ and $x$, and backpropagate the gradient optimization model. In order to achieve the color transformation for the facial skin area and the eye area, we only use histogram loss on these two areas, $L_{G}^{\text {local }}$ is as follows:

$$
\begin{gathered}
L_{G}^{\text {local }}=\lambda_{s}\left[\left\|G(x, y)-H M\left(G(x, y)^{\circ} M_{\text {skin }}^{1}, y^{\circ} M_{\text {skin }}^{2}\right)\right\|_{2}\right] \\
+\lambda_{e}\left[\left\|G(x, y)-H M\left(G(x, y)^{\circ} M_{\text {eye }}^{1}, y^{\circ} M_{\text {eye }}^{2}\right)\right\|_{2}\right] \\
M^{1}=F P(G(x, y)) \\
M^{2}=F P(y)
\end{gathered}
$$

where $\lambda_{s}$ and $\lambda_{e}$ represent the weight.

\subsection{Adversarial Learning}

For the non-sick face image data domain $\mathrm{X}$ and the sick face image data domain $\mathrm{Y}$, our network uses two discriminators $\mathrm{D}_{\mathrm{X}}$ and $\mathrm{D}_{\mathrm{Y}}$ respectively. The discriminator is used to distinguish the generated image and the real sample, so that the generator can output real results. The objective function of the discriminator only contains the adversarial loss. The adversarial losses $\mathrm{L}_{\mathrm{D}_{\mathrm{X}}}^{\mathrm{adv}}$ and $\mathrm{L}_{\mathrm{D}_{\mathrm{Y}}}^{\mathrm{adv}}$ of the discriminators $\mathrm{D}_{\mathrm{X}}$ and $\mathrm{D}_{\mathrm{Y}}$ are as follows:

$$
\begin{gathered}
L_{D_{X}}^{a d v}=E_{x \sim p_{\text {data }}(x)}\left[\log D_{X}(x)\right] \\
+E_{x \sim p_{\text {data }}(x), y \sim p_{\text {data }}(y)}\left[\log \left(1-D_{X}(G(y, x))\right]\right. \\
L_{D_{Y}}^{a d v}=E_{y \sim p_{\text {data }}(y)}\left[\log D_{Y}(y)\right] \\
+E_{x \sim p_{\text {daza }}(x), y \sim p_{\text {data }}(y)}\left[\log \left(1-D_{Y}(G(x, y))\right]\right.
\end{gathered}
$$

Therefore, the adversarial loss of generator $G$ is as follows:

$$
L_{G}^{a d v}=L_{D_{X}}^{a d v}+L_{D_{T}}^{a d v}
$$

\subsection{Cycle Consistency}

In order to prevent the network from generating random useless images in the target data domain under unsupervised learning, we introduce a cyclic consistency constraint. For each input picture $x$ in the data domain $X$, an image similar to it should be generated after the image is cyclically translated, that is, $x \rightarrow G(x, y) \rightarrow$ $G(G(x, y)) \approx x$. We use L1 loss to constrain the reconstructed image, and the cyclic consistency loss $L_{G}^{c y c}$ is shown in the following formula:

$$
\begin{gathered}
L_{G}^{c y c}=E_{x \sim p_{\text {dat }}(x), y \sim p_{\text {dat }}(y)}\left[\|G(G(x, y), x)-x\|_{1}\right] \\
+E_{x \sim p_{\text {daz }}(x), y \sim p_{\text {data }}(y)}\left[\|G(G(y, x), y)-y\|_{1}\right]
\end{gathered}
$$

\subsection{Perceptual Loss}

When performing sick feature transfer on the source image, the generated image should keep the primary information such as age, gender, and face shape unchanged. Different from directly measuring the difference at the pixel level, we extract high-level features through a VGG-16 model pre-trained on the ImageNet and compare the input image's activation values and the generated image on the hidden layer. We use L2 loss to measure the difference between the input image and the generated image. The perceptual loss $L_{G}^{\text {per }}$ is shown in the following formula:

$$
\begin{aligned}
& L_{G}^{\text {per }}=E_{x \sim p_{\text {data }}}(x), y \sim p_{\text {data }}(y)\left[\| F_{l}\left(G(x, y)-F_{l}(x) \|_{2}\right]\right. \\
& \quad+E_{x \sim p_{\text {data }}(x), y \sim p_{\text {data }}(y)}\left[\| F_{l}\left(G(y, x)-F_{l}(y) \|_{2}\right]\right.
\end{aligned}
$$

where $F_{l}(\cdot)$ represents the feature map of the lth layer of the VGG model.

\subsection{Objective Function}

The objective function of the generator can be expressed as:

$$
L_{G}=\lambda_{a d v} L_{G}^{a d v}+\lambda_{c y c} L_{G}^{c y c}+\lambda_{p e r} L_{G}^{p e r}+\lambda_{\text {local }} L_{G}^{\text {local }}
$$

where $\lambda_{a d v}, \lambda_{c y c}, \lambda_{\text {per }}$ and $\lambda_{\text {local }}$ respectively represent the weight of each loss term.

\section{EXPERIMENTS}

\subsection{Data Collection}

We construct a sick face image dataset. The dataset has 3046 face images, including 927 sick face images and 2119 non-sick face images. The sick face images in the dataset have varying degrees of dark circles and dull complexions, while the non-sick face images are healthy faces that do not contain noticeable sick features or have been modified by technical means such as beauty filters and makeup. The face images we have collected contain different poses, expressions, and backgrounds. Face data is initially crawled from the Internet, and then images with low resolution and poor lighting conditions are manually removed. We perform key point detection on each picture in the sick face image dataset, use 68 facial landmarks for face alignment, and crop the image size to $256 \times 256$ according to the two eyes' positions. We randomly select 150 sick face images and 100 non-sick face images from the dataset for testing. The remaining images are divided into a training set and a validation set, and the ratio of the two groups is 10:1.

\subsection{Implementation Details}

For all experiments, we use PSPNet [21] to perform semantic segmentation on face images to obtain mask labels with different areas of the face. In addition, we use the relu_4_1 feature layer in VGG16 pre-trained on ImageNet to calculate the perceptual loss. The weights of each loss function of the network are respectively set as: $\lambda_{a d v}=1, \lambda_{c y c}=10, \lambda_{\text {per }}=0.005, \lambda_{\text {local }}=1, \lambda_{f}=0.1, \lambda_{e}=1$. We use Adam to train the network, the learning rate is set to 0.0002 , and the batch size is set to 1 .

\subsection{Ablation Studies}

In PathosisGAN, the mask control module generates a binary mask in the facial skin area and the eye area. And then, we use histogram loss for these two areas, respectively, thereby transferring the dark circles and dull complexion of the sick face image to the source image and maintain consistency in irrelevant areas. To demonstrate the effectiveness of the mask control module, we perform 3 experiments. The results are shown in Figure 4. Experiment 1 removes the histogram loss of facial skin. The third column shows the generated images are slightly modified on skin color with noticeable dark circles. In Experiment 2, the histogram loss term of eye area is removed. We find the generated images (the fourth column in 


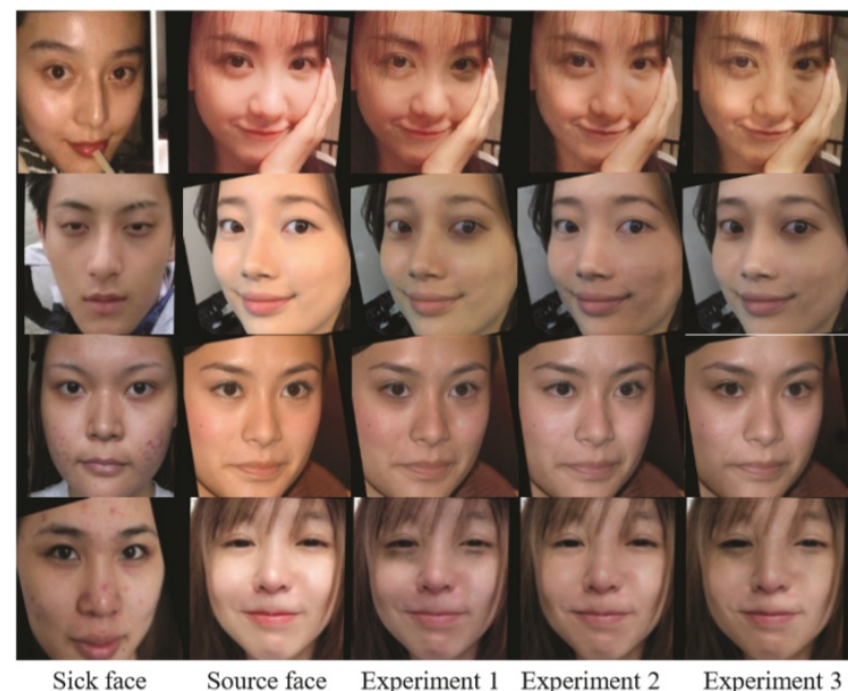

Figure 4: Results of ablation study. The first two columns are sick face images and source images respectively. Each row in the remaining columns shows the results of Experiment 1, Experiment 2 and Experiment 3.

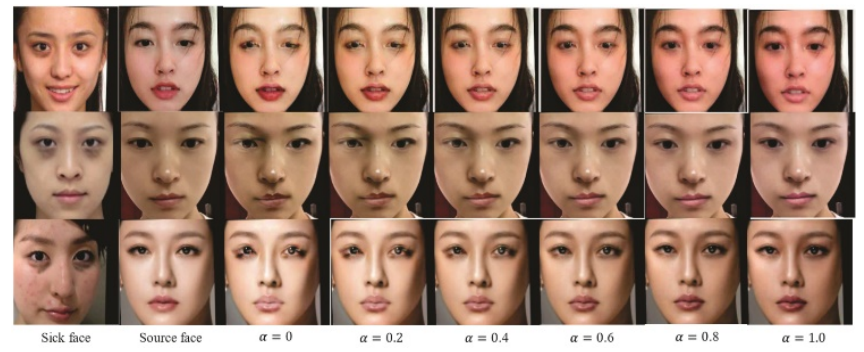

Figure 5: The first two columns are sick face images and source images respectively. Each row in the remaining columns shows the results with controllable degrees of sickness.

Figure 4) do not transfer dark circles from the sick face images, only the skin color is changed. Experiment 3 has the histogram loss terms of both eye area and the facial skin. We observe that the generated images (the fifth column in Figure 4) successfully transfer the dark circles and skin color from the sick face images to the source images.

To sum up, the histogram loss terms of different areas can be used to edit the corresponding areas independently, and restrain the changes of irrelevant areas, which can be used to realize the transfer of specific sick features.

In the feature extraction module, the FDNet extracts two feature matrixes $\psi$ and $\omega$ from the image feature map, and the feature matrixes are repeatedly expanded along with the number of channels into feature tensors $\psi$ and $\Omega$, synthesizes face images with different degrees of sickness by adjusting the weight $\alpha \in[0,1]$. The generated results in Figure 5 show that PathosisGAN can effectively
Table 1: Results of user study

\begin{tabular}{lccl}
\hline Methods & Rank1 & Rank2 & Rank3 \\
\hline CycleGAN & $32.79 \%$ & $48.63 \%$ & $18.58 \%$ \\
DIA & $8.72 \%$ & $15.65 \%$ & $75.63 \%$ \\
PathosisGAN & $58.49 \%$ & $35.72 \%$ & $5.79 \%$ \\
\hline
\end{tabular}

adjust the degree of dark circles from mild to severe, and make the complexion gradually worse.

\subsection{Comparison}

This paper adopts CycleGAN [6] and DIA [22], two effective imageto-image translation methods as baselines. CycleGAN is a typical method of image-to-image translation under unsupervised learning. It translates the image from the source data domain to the target data domain in the absence of paired data. DIA is used to process images with different appearances but similar semantic structures. It matches the features extracted from the deep Convolutional Neural Network and transfers visual information from one image to another. We compare PathosisGAN with the above two types of methods from both qualitative and quantitative dimensions.

4.4.1 Qualitative Comparison. We conducted a user study from 5 volunteers for quantitative comparison. We randomly selected 20 sick face images and 20 source images from the dataset. After using CycleGAN, DIA and PathosisGAN to perform sick face image synthesis between these images, we obtain 800 images for each method. We randomly shuffled the results generated from different methods. Participants are asked to rank the three generated images considering the quality, realism, and clarity of the pathological features of the generated images. Rank 1 means the best effect, and rank 3 means the least. We use the percentages of the three rankings for each method as the result of quantitative comparison. As shown in the Tab.1, we observe that most of the generated results of PathosisGAN rank one and its performance is significantly better than the other two benchmark methods.

4.4.2 Qualitative Comparison. Figure 6 indicates the results of qualitative comparison results with baselines. CycleGAN can synthesize realistic images, but the synthesized images have no noticeable skin color changes and dark circles. Although there are some slight differences in skin color and eye area of DIA synthesized result, we find that the color of the background area of the synthesized image has also changed, and the degree of sickness (especially the dark circles) of the synthesized image is much weaker than that of the input sick face image. When the input image's face size and facial expression are greatly different, the generated face image will be distorted. Consequently, the visual effect is poor.

Compared with the above methods, PathosisGAN can modify the local area of the face, and make sure that the areas that do not need to be edited keeping unchanged at the same time, such as hair and background.

For better demonstrating the comparison, we zoom in the performance of dark circles and dull complexion transfer. From the detailed images of the eyes and facial skin in Figure 7, it is not difficult to see that the complexion changes and dark circles of the 


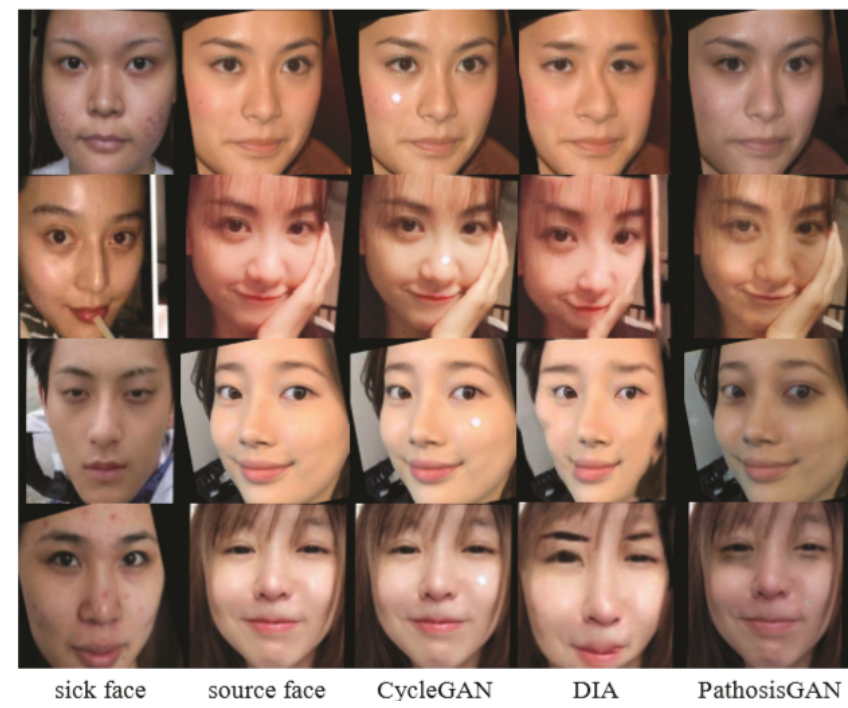

Figure 6: Qualitative comparison between PathosisGAN and baselines. The first two columns are sick face images and source images respectively. Each row in the remaining columns shows the generated images by different methods.
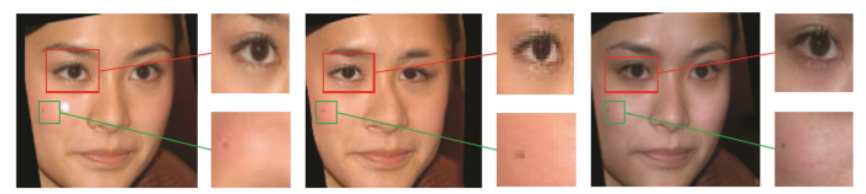

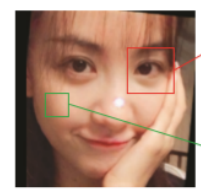

CycleGAN
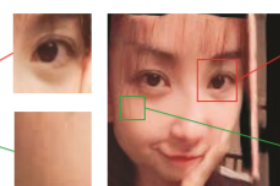

DIA
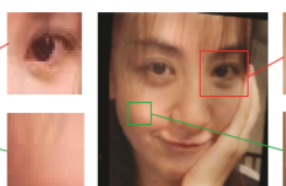

PathosisGAN
Figure 7: Zoom in the performance of dark circles and dull complexion transfer.

synthesized image using PathosisGAN are the most noticeable and visually more natural.

\section{CONCLUSION}

In this paper, we propose a novel Generative Adversarial Network model called PathosisGAN, which realizes sick face image synthesis under unsupervised learning. We introduce the mask control module to successfully transfer the sick features in the local area of the sick face to the source image and retain other source images primary information. In addition, we add a feature extraction module to the generator. The feature extraction module can extract feature tensors from the feature map to synthesize faces with different degrees of sickness. The experimental results show that our method performs well in synthesizing sick face images, and effectively expands the sick face image dataset. For future research, we will further investigate the sick facial feature recognition with PathosisGAN-based data augmentation methods.

\section{ACKNOWLEDGMENTS}

The author would like to thank the reviewers of this conference for their hard work. The work is supported by the National Natural Science Foundation of China (Grant No.31871521).

\section{REFERENCES}

[1] Y. Taigman, M. Yang, M. Ranzato, and L. Wolf. Deepface: Closing the gap to human-level performance in face verification. In CVPR, vol.1. 2014.

[2] R. Girshick. Fast r-cnn. In ICCV, vol.1. 2015

[3] G .B. Huang, M. Mattar, T. Berg, and E. Learned-Miller. Labeled faces in the wild: A database forstudying face recognition in unconstrained environments. In ECCV, vol. 1. 2008.

[4] I. Goodfellow, J. Pouget-Abadie, M. Mirza, B. Xu, D. Warde-Farley, S. Ozair, A. Courville, and Y. Bengio. Generative adversarial nets. In NIPS, vol. 1, no. 2. 2014.

[5] P.Isola,J.-Y.Zhu,T.Zhou,andA.A.Efros. Image-to-image translation with conditional adversarial networks. In CVPR, vol. 1, no. 2. 2017

[6] J.-Y.Zhu, T. Park, P. Isola, and A. A.Efros. Unpaired image-to-image translation using cycle-consistent adversarial networks. In ICCV, 2017. 1, 2, 6

[7] T. Kim, M. Cha, H. Kim, J. K. Lee, and J. Kim. Learning to discover cross-domain relations with generative adversarial networks. In ICML, vol. 1, no. 2. 2017.

[8] Z. Yi, H. Zhang, P. Tan, and M. Gong. Dualgan: Unsupervised dual learning for image-to-imagetranslation. InICCV, vol. 1. 2017.

[9] M. Li, W. Zuo, and D. Zhang. Deep identity-aware transfer of facial attributes. arXiv preprint arXiv:1610.05586, 2016.

[10] W. Shen and R. Liu. Learning residual images for face attribute manipulation. In CVPR, vol. 2. 2017.

[11] Z. Zhang, Y. Song, and H. Qi. Age progression/regression by conditional adversarial autoencoder. In CVPR, vol. 2. 2017.

[12] Y. Lu, Y.-W. Tai, and C.-K. Tang. Conditional cyclegan for attribute guided face image generation. arXiv preprint arXiv:1705.09966, vol. 2. 2017.

[13] C. Wang, C. Xu, C. Wanga, and D. Tao. Perceptual adversarial networks for image-to-image transformation. IEEE Transactions on Image Processing, vol. 2. 2018.

[14] T. Kim, B. Kim, M. Cha, and J. Kim. Unsupervised visual attribute transfer with reconfigurable generative adversarial networks. arXiv preprint arXiv:1707.09798, 2017.

[15] C. Shorten and T. M. Khoshgoftaar. A survey on image data augmentation for deep learning. Journal of Big Data, vol. 2. 2019.

[16] X. Yi, E. Walia, and P. S. Babyn. Generative adversarial network in medical imaging: A review. Medical Image Analysis, vol. 2. 2019.

[17] J. M. Wolterink, T. Leiner, M. A. Viergever, and I. Isgum. Generative adversarial networks for noise reduction in lowdose ct. IEEE Transactions on Medical Imaging, vol. 2. 2017.

[18] M. Frid-Adar, I. Diamant, E. Klang, M. Amitai, J. Goldberger, and H. Greenspan. Gan-based synthetic medical image augmentation for increased cnn performance in liver lesion classification. Neurocomputing, vol. 2. 2018.

[19] S. W. Huang, C. T. Lin, S. P. Chen, Y. Y. Wu, and S. H. Lai. Auggan: Cross domain adaptation with gan-based data augmentation. In ECCV, vol. 2. 2018.

[20] K. Ehsani, R. Mottaghi, and A. Farhadi. Segan: Segmenting and generating the invisible. In CVPR, vol. 2. 2018.

[21] H. Zhao, J. Shi, X. Qi, X. Wang, and J. Jia. Pyramid scene parsing network. In CVPR, vol. 5. 2017.

[22] J. Liao, Y. Yao, L. Yuan, G. Hua, and S. B. Kang. Visual attribute transfer through deep image analogy. Acm Transactions on Graphics, vol. 6. 2017. 